\title{
Endobronchial actinomycosis in a child during COVID-19 pandemic
}

Pierre Goussard ${ }^{1}$, Helena Rabie ${ }^{2}$, Michelle Marshall ${ }^{2}$, Lunga Mfingwana ${ }^{2}$, Julie Morrison ${ }^{2}$, Zane Ismail ${ }^{2}$, Francois Retief ${ }^{2},{\text { Pieter } \mathrm{Nel}^{2} \text {, Dawood Dacosta }}^{2}$, Savvas Andronikou ${ }^{3}$, and Pawel Schubert ${ }^{2}$

${ }^{1}$ Stellenbosch University

${ }^{2}$ Stellenbosch University Faculty of Medicine and Health Sciences

${ }^{3}$ University of Pennsylvania

May 8, 2021

\begin{abstract}
Actinomycosis is a rare, indolent and invasive infection caused by Actinomyces species. Pulmonary actinomycosis is very rarely seen in the paediatric population. The classic radiological presentation of thoracic involvement of actinomycosis includes lower lobe consolidation, empyema and periostitis of the ribs. We report a case of endobronchial pulmonary actinomycosis in a child diagnosed on endobronchial biopsy and broncho-alveolar lavage taken during bronchoscopy. Bronchoscopy can be dangerous when performed on these cases, as there is a risk of severe bleeding and large airway obstruction, as was the case with this patient.
\end{abstract}

\section{Endobronchial actinomycosis in a child during COVID-19 pandemic}

Goussard $\mathrm{P}^{1}(\mathrm{PhD})$, Rabie $\mathrm{H}^{1}(\mathrm{PhD})$, Marshall $\mathrm{M}^{2}$ (MSc Pathology), Mfingwana $\mathrm{L}^{1}$ (FC Paed(SA)), Morrison $\mathrm{JL}^{1}$ (FC Paed(SA)), Ismal Z $\mathrm{Z}^{3}$ (FC Cardio (SA)), Retief $\mathrm{F}^{4}$ (MMed Anaesthesiology), Nel $\mathrm{P}^{5}$ (FC Path Micro (SA)), Da Costa $D^{5}(\mathrm{MBChB})$ Andronikou $\mathrm{S}^{6}(\mathrm{PhD})$, Schubert $\mathrm{P}^{7}$ MPhil (Paed Path))

${ }^{1}$ Department of Paediatrics and Child Health, Faculty of Medicine and Health Sciences, Stellenbosch University, Tygerberg Hospital, Cape Town, South Africa.

${ }^{2}$ Division of Cytology, Tygerberg Hospital, National Health Laboratory Service, Cape Town, South Africa.

${ }^{3}$ Division of Cardiothoracic Surgery, Department of Surgical Sciences, Faculty of Medicine and Health Sciences, Stellenbosch University, Tygerberg Hospital, Cape Town, South Africa.

${ }^{4}$ Department of Anaesthesiology and Critical Care, Faculty of Medicine and Health Sciences, Stellenbosch University, Tygerberg Hospital, Cape Town, South Africa

${ }^{5}$ Division of Medical Microbiology, Department of Pathology, Faculty of Medicine and Health Sciences, Stellenbosch University and National Health Laboratory Service, Tygerberg Hospital, Cape Town, South Africa

${ }^{6}$ Department of Paediatric Radiology, The Children's Hospital of Philadelphia and University of Pennsylvania, Philadelphia, PA, United States of America.

${ }^{7}$ Division of Anatomical Pathology, Department of Pathology, Faculty of Medicine and Health Sciences, Stellenbosch University and National Health Laboratory Service, Tygerberg Hospital, Cape Town, South Africa. 
Address for correspondence: Prof Pierre Goussard, Department of Paediatrics and Child Health, Faculty of Medicine and Health Sciences, Stellenbosch University, PO Box 241, Cape Town 8000, South Africa. Tel +27-21-938-9506; Fax +27-21-938-9138; Email pgouss@sun.ac.za

Keywords: Endobronchial actinomycosis, bronchoscopy, dental caries, tuberculosis, sulphur granules.

\section{Introduction}

Actinomycosis is a rare, indolent and invasive infection caused by Actinomyces species. These facultative anaerobic bacteria are constituents of the normal flora of the oropharynx, gastrointestinal tract and female genital tract. Actinomycosis develops when there is disruption of the mucosal barrier, invasion and systemic spread of the organism, which can lead to endogenous infection affecting numerous organs.

Actinomyces is known to spread in tissue through fascial planes and most often involves the cervicofacial (55\%), abdominopelvic (20\%) and thoracic (15\%) soft tissue. Nevertheless the skin, central nervous system, pericardium and bone can also be affected. [1,2] Risk factors for actinomycosis in children include dental caries, trauma, debilitation, poorly controlled diabetes mellitus and foreign body aspiration. [3]

Pulmonary actinomycosis, very rarely seen in the paediatric population, can present with cough, fever, haemoptysis and weight loss. The classic radiological presentation of thoracic involvement of actinomycosis includes lower lobe consolidation, empyema and periostitis of the ribs. $[4,5]$ We report a case of endobronchial pulmonary actinomycosis in a child diagnosed on endobronchial biopsy, taken during bronchoscopy.

\section{Case Presentation}

An eight-year-old HIV-negative boy presented with a history of chronic cough and weight loss over a one-year period. He had no known adult tuberculosis (TB) contacts. During this time he was seen at his local clinic on three occasions and received a five-day course of oral amoxicillin on two of these visits.

His mother reported that he also complained of tooth ache but decided to forego an assessment by the dentist. On examination his body mass index was 18.5 (normal WHO Z score) and he had clubbing of the fingers and toes. Severe dental caries were observed. On respiratory examination, his chest wall was normal, but there was dullness to percussion in the right middle and lower lobe areas.

His Mantoux skin test was negative.

Antero-posterior and lateral chest X-rays (Figure $1 \mathrm{~A}$ and B) performed on admission demonstrated cutoff of the bronchus intermedius with dense consolidation of the right lower and middle lobes. Contrasted computed tomography (CT) scans of the chest (Figure $1 \mathrm{C}-\mathrm{E}$ ) performed four days after the plain radiographs, confirmed abrupt narrowing of the bronchus intermedius as well as dense air-space disease in the right lower and middle lobes, with a large confluent area of central low density lacking parenchymal structures and vessels indicative of necrosis without gas formation. Further features included a small effusion at the right base, and large non-necrotic, non-enhancing lymph nodes in the superior mediastinal, right paratracheal, subcarinal and hilar areas.

Bronchoscopy was performed with a $4.9 \mathrm{~mm}$ scope with a $2.2 \mathrm{~mm}$ working channel (Olympus BF-H1100 Broncho videoscope). There was complete obstruction and narrowing of the bronchus intermedius (Figure $1 \mathrm{~F})$. Yellow, solid-appearing material was present in the bronchus intermedius with only the right upper lobe bronchus patent. A biopsy of the material was taken and the obstruction was relieved by meticulously removing the adherent soft material. Once the distal part of the right lower lobe bronchus could be reached, the presence of foreign bodies and tumours were excluded. Due to significant bleeding, the procedure had to be interrupted several times to allow for effective ventilation.

Following biopsy and clearing of puss and blood by suctioning through the bronchoscope, a $5.5 \mathrm{~mm}$ cuffed endotracheal tube (ETT) was placed in the trachea. Despite ventilation pressures of 30-40 $\mathrm{mmH} 2 \mathrm{O}$, there was very little chest expansion which indicated further obstruction. A $5 \mathrm{~cm}$ long yellow mass clot, too thick to pass 
through the bronchoscope working channel, was removed with an suction catheter after which ventilation returned to normal.

The tissue and broncho-alveolar lavage (BAL) specimens were sent for a full laboratory workup including cytology, histology, microbiology and virology.

The cytology on BAL showed clumps of amorphous material in which red filamentous organisms could be seen. A Periodic acid-Schiff (PAS) and Brown-Hopps Gram stains revealed these to be gram-positive filamentous bacilli which morphologically suggested Actinomyces species (Figure $1 \mathrm{G}-\mathrm{L}$ ). The differential count on the BAL fluid was as follows: macrophages: 0\%, lymphocytes: $4 \%$, neutrophils: $96 \%$, and eosinophils: $0 \%$.

The histology showed necrotic tissue that had a slight fibrillary character. There was a focal areas which showed suggestion of a Splendore-Hoeppli effect with the filaments lining up close to the surface and interacting with inflammatory cells.

Brown-Hopps stain showed gram-positive filamentous organisms which were Ziehl-Neelsen (ZN) negative, PAS positive and negative on Kinyoun acid-fast stain (Figure $\mathrm{K}$ and $\mathrm{L}$ ). The Gram stain showed more than 20 polymorphonuclear leucocytes per low power field, with abundant filamentous gram-positive bacilli (Figure M).

This morphology together with the clinical information, would indicate an organism ball and sulphur granule. The filamentous nature of these bacteria, together with being $\mathrm{ZN}(-)$ and $\mathrm{PAS}(+)$ is highly suggestive of actinomycosis.

Bacterial culture produced a mixed growth and, despite 14 day aerobic and anaerobic incubation, failed to isolate Nocardia or Actinomyces . The Xpert ${ }^{\circledR}$ MTB RIF/Ultra (Cepheid, CA, USA), TB culture and the respiratory multiplex PCR was also negative.

The child's immunological work-up was normal. He was treated for 14 days with intravenous amoxicillin/clavulanic acid and then changed to oral amoxycillin and he was referred to a dentists who removed the affected teeth. At a 3 month follow-up visit he had significant clinical and radiological improvement. He will be followed up regularly to decide if surgical intervention is necessary and will oral amoxycillin therapy for at least 12 months .

\section{Ethical considerations}

Consent was given by the patient's parents for publication of this case and the report.

\section{Discussion}

Actinomyces species were first identified in Israel during 1878 from human necropsy tissue. [6] Ponfick reported the first case of thoracic actinomycosis in 1882. [7]

Several species of Actinomyces have been identified in humans with at least 21 species identified with the use of $16 \mathrm{~S}$ rRNA sequencing. [8] Actinomyces israelii remains the predominant species causing cervicofacial, abdominopelvic, and thoracic actinomycosis.

Diagnosis of paediatric pulmonary actinomycosis remains a challenge because it is a rare disease (even in resource limited settings) with no specific features on imaging. CT findings that have been described include airspace consolidation, lobar consolidation, ground glass opacification, pleural effusions, pleural thickening, hilar lymphadenopathy and a necrotic mass. [9] Both the CXR and CT-scan findings may mimic TB, which can lead to misdiagnosis in settings with a high TB burden. In addition to the diagnostic difficulties, this patient experienced a delay in referral for nearly a year due to the SARS-CoV-2 pandemic. Diagnosis was further delayed due to limited chest x-rays during the pandemic.

In cases of endobronchial involvement, a follow-up bronchoscopy is essential after treatment has started and imaging has shown improvement in order to exclude a foreign body. Foreign body-induced endobronchial 
actinomycosis have been reported in $45 \%$ of adult cases and in most cases the foreign body was detected only after starting antibiotic therapy. In these cases, the foreign body should always be removed. [10]

Patients with thoracic actinomycosis may be candidates for individualized courses of therapy depending on the initial burden of disease, whether resection surgery was performed, and the clinical and radiologic responses to therapy. The traditional recommendation of IV antibiotic therapy for 2 to 6 weeks followed by oral antibiotic therapy for 6 to 12 months is not always necessary. [11]

\section{Conclusion}

We present a rare case of advanced endobronchial actinomycosis in a child. We postulate that dental caries were the source of the infection, which led to micro aspiration of the pathogen. We postulate that there was delay in referral and diagnosis due to the SARS-CoV-2 pandemic. . This case highlights the difficulty in diagnosis of rare lung conditions in high incidence settings of tuberculosis, particularly where there is limited access to specialised pulmonology, infectious diseases and microbiology services. The diagnosis in this case was made on BAL. Bronchoscopy can be dangerous when performed on these cases, as there is a risk of severe bleeding and large airway obstruction, as was the case with this patient.

\section{Reference}

1. Hsieh MJ, Liu HP, Chang JP, Chang CH. Thoracic actinomycosis. Chest. 1993 Aug;104(2):366-70. doi: 10.1378/chest.104.2.366. PMID: 8339619.

2. Thompson AJ, Carty H. Pulmonary actinomycosis in children. Pediatr Radiol. 1979 Feb 26;8(1):7-9. doi: 10.1007/BF00973668. PMID: 431995.

3. Bartlett AH, Rivera AL, Krishnamurthy R, Baker CJ. Thoracic actinomycosis in children: case report and review of the literature. Pediatr Infect Dis J. 2008 Feb;27(2):165-169. doi: 10.1097/INF.0b013e3181598353. PMID: 18174864.

4. Kirklin BR, Hefke HW. The roentgenologic aspects of actinomycosis of the lungs. Am J Surg 1931;13:18. https://doi.org/10.1016/S0002-9610(31)90530-8

5. Flynn ME, Felson B. The roentgen manifestations of thoracic actinomycosis. Am J Roentgenol Radium Ther Nucl Med 1970;110: 707-716. 10.2214/ajr.110.4.707.

6. Israel J. Neue Beobachtungen auf dem Gebiete der Mykosen des Menschen. Virchows Arch A Pathol Anat 1878;74:15-53.

7. Ponfick E. Die Actinomykose des Menschen eine neue Infectionskrankheit auf vergleichendpathologischen und experimenteller Grundlage geschildert. Berlin: Hirschwald; 1882.

8. Clarridge III JE, Zhang Q. Genotypic diversity of clinical Actinomyces species: phenotype, source, and disease correlation among genospecies. J Clin Microbiol 2002;40(9):3442-3448. doi:10.1128/jcm.40.9.3442-3448.2002.

9. Kim TS, J, Won-Jung Koh WJ, Choi JC, Chung MJ, Lee JH, Shim SS, Chong S. Thoracic actinomycosis: CT features with histopathologic correlation. AJR. American Journal of Roentgenology. 2006 Jan;186(1):225-231. DOI: 10.2214/ajr.04.1749.

10. Chouabe S, Perdu S, Deslee G, Milosevic D, Marque E, Lebargy F. Endobronchial actinomycosis associated with foreign body: four cases and a review of the literature. Chest 2002;121(6):2069-2072. doi: 10.1378/chest.121.6.2069. PMID: 12065381.

11. Choi J, Koh WJ, Kim TS, Lee KS, Han J, Kim H, Kwon OJ. Optimal duration of IV and oral antibiotics in the treatment of thoracic actinomycosis. Chest. 2005 Oct;128(4):2211-7. doi: 10.1378/chest.128.4.2211. PMID: 16236876.

\section{Legends}

\section{Figure 1 A-M}

(A) \& (B) Frontal and lateral radiographs in an 8-year-old boy demonstrate cut-off of the bronchus intermedius (arrows) and the confluent air-space process in the right lower and middle lobes

(C)-(E) Post IV contrast CT scan of the chest: 
(C) Axial slice on soft tissue window at the subcarinal level demonstrates airspace disease with airbronchograms and enhancing lung tissue (star), foci of non-enhancement (white arrows) indicating non-viable lung tissue as well as subcarinal lymphadenopathy (black arrow) and narrowing of the bronchus intermedius.

(D) Axial slice on soft tissue window at the level of the right base demonstrates a confluent area of necrosis (star) without visible lung structures, which does not contain gas and measures $15 \mathrm{HU}$, in keeping with complex necrotic fluid material. A small effusion is appreciated medial to the right crus of the diaphragm.

(E) Coronal Minimum Intensity Projection (MinIP) reconstruction angles along the airway demonstrates the cut-off of the bronchus intermedius (arrow) and dense right basal consolidation.

(F) Bronchoscopy images: Bronchoscopy images of bronchus intermedius demonstrating complete obstruction of the airway with a yellow appearing lesion with external compression of the airway.

(G) Bronchoalveolar lavage showing a small sulphur granule in a rather degenerate background (pap stain 200x).

(H) Bronchoalveolar lavage showing a higher magnification image of the sulphur granule being made up of numerous entangled filamentous bacteria.

(I) Biopsy of the bronchial mucosal sulphur granule showing a small granule surrounded by neutrophils and condensation of the bacteria on the surface being highlighted as a darker staining colour (H\&E stain, 200x).

(J) Higher magnification showing the granule being composed of filamentous bacteria, similar to Figure $1 \mathrm{H}$ (H\&E stain, 1000x).

(K) Brown-Hopps stain highlighting these bacteria as gram-positive. (Brown-Hopps gram, 1000x).

(L) PAS stain also staining the bacteria (PAS stain, 1000x)

(M) Gram stain showing abundant polymorphonuclear leucocytes, and filamentous gram-positive bacilli (Gram stain, 100x)

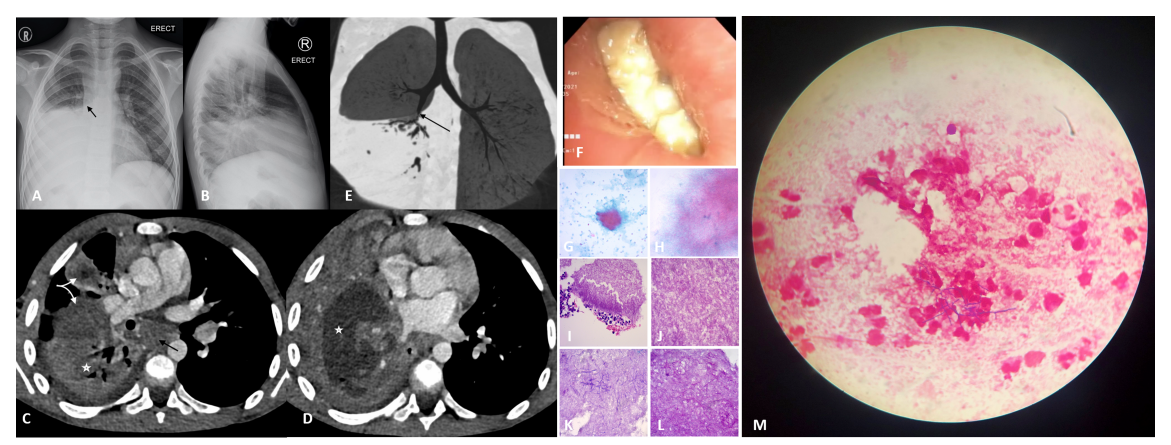

